apuntesuniversitarios.upeu.edu.pe

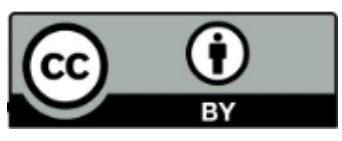

$C C-B Y$

Apuntes Universitarios, 2021: 11(4), octubre-diciembre

ISSN: 2304-0335 DOI:https://doi.org/10.17162/au.v11i4.759

\title{
Procrastinación académica: un estudio descriptivo con estudiantes de secundaria
}

\author{
Academic procrastination: a descriptive study with \\ high school students
}

\begin{abstract}
Gilber Chura Quispe, ${ }^{1 a}$ Sugey Milagros Calderón Carazas, ${ }^{2}$ María Sonia Castro Marrón, ${ }^{3}$ Amparo Verástegui Quintanilla ${ }^{4}$
\end{abstract}

Universidad Nacional Jorge Basadre Grohmann, Tacna, Perú ${ }^{1234}$

(iD) ORCID ID: https://orcid.org/0000-0002-3467-2695 ${ }^{1}$

(iD) ORCID ID: https://orcid.org/0000-0003-0847-684X ${ }^{2}$

(iD) ORCID ID: https://orcid.org/0000-0002-5764-8129 3

(iD) ORCID ID: https://orcid.org/0000-0002-2981-1902 4

Recibido: 22 de febrero de 2021

Aceptado: 28 de mayo de 2021

\begin{abstract}
Resumen
El objetivo del estudio fue identificar el nivel de procrastinación académica de los estudiantes de educación secundaria de Tacna en la educación a distancia. El enfoque fue cuantitativo, con diseño no experimental y tipo descriptivo-transversal. La muestra estuvo constituida por 338 estudiantes de secundaria. El instrumento empleado fue la "Escala de Procrastinación Académica (EPA)" con adecuados índices de validez y confiabilidad. Los resultados muestran que, en la postergación de actividades, la mayoría de estudiantes presenta un nivel promedio (54 \%) y en la autorregulación académica es alto (49.70\%), pero una cuarta parte mostró bajos niveles $(24.85 \%)$. También, se encontró diferencia entre los grados de estudios $(\mathrm{p}=0.013$ y 0.001$)$ y los estudiantes hombres $(\mathrm{p}=$ 0.000 y 0.000$)$, de instituciones privadas ( $\mathrm{p}=0.000$ y 0.002 ) y quienes no se autoconsideran buenos académicamente ( $\mathrm{p}=0.000$ y 0.000 ), que tienden a postergar más sus actividades y a mostrar menor autorregulación académica. Se concluyó que no predomina un alto nivel de procrastinación en los estudiantes, pero sí existen diferencias significativas entre las variables sociodemográficas.
\end{abstract}

Palabras clave: Procrastinación académica, educación secundaria, autorregulación académica, educación a distancia

${ }^{a}$ Correspondencia al autor:

E-mail: gilber.chura@unjbg.edu.pe 


\begin{abstract}
The objective of the study was to identify the level of academic procrastination of secondary school students in Tacna in distance education. The approach was quantitative, with a non-experimental design and descriptive-transversal type. The sample consisted of 338 secondary school students. The instrument used was the "Academic Procrastination Scale (EPA)" with adequate validity and reliability indexes. The results show that, in the procrastination of activities, most students present an average level (54\%) and in academic self-regulation it is high (49.70\%), but a quarter showed low levels $(24.85 \%)$. There is a difference between grade levels $(\mathrm{p}=0.013$ and 0.001$)$ and, in addition, male students ( $\mathrm{p}=0.000$ and 0.000$)$, students from private institutions $(\mathrm{p}=0.000$ and 0.002$)$ and those who do not consider themselves academically good ( $\mathrm{p}=0.000$ and 0.000$)$ tend to procrastinate more and show less academic self-regulation. It was concluded that a high level of procrastination does not predominate among students, but there are significant differences among sociodemographic variables.
\end{abstract}

Keywords: Academic procrastination, secondary education, academic self-regulation, distance education

\title{
Introducción
}

El impacto del confinamiento social por causa del COVID-19 ha generado cambios sociales y económicos a nivel nacional e internacional. En el caso de la educación, la modalidad de estudio ha pasado de la estancia presencial a la virtual y, de esta forma, las brechas educativas se han incrementado debido a la falta de recursos tecnológicos, principalmente en países en vías de desarrollo. De acuerdo con la Organización de las Naciones Unidas para la Educación, Ciencias y Cultura (UNESCO), el cierre de escuelas afecta a más de 800 millones de estudiantes, que representa el $50 \%$ de matriculados (Organización de las Naciones Unidas para la Educación la Ciencia y la Cultura, 2020). Ante esta situación, los gobiernos, ministerios e instituciones que organizan la educación en cada país han tomado medidas acordes a sus realidades.

En el caso de la educación pública peruana, de acuerdo con la R.M. º 160-2020-MINEDU, desde el 06 de abril de 2020 se ha propuesto la educación remota bajo la estrategia «Aprendo en casa» a través de radio, televisión y plataforma virtual (Ministerio de Educación, 2020b). De la misma forma, para la educación privada, la R.V. $\mathrm{N}^{\circ}$ 079-2020-MINEDU ha dispuesto la reprogramación de las horas lectivas educativas tomando en consideración las características y necesidades del aprendizaje de los escolares (Ministerio de Educación, 2020a). Es así que el proceso de enseñanza-aprendizaje en ambos tipos de educación sigue líneas distintas por las condiciones socioeconómicas de la comunidad educativa y visión de las instituciones. 
Aunque las modalidades educativas (virtual y a distancia) se diferencian por el empleo de herramientas tecnológicas, existe ciertos problemas pedagógicos que persisten en la educación como el gusto por el trabajo bajo presión, intención de posponer actividades, bajo rendimiento académico, empleo de internet o desinterés por iniciar una tarea (Suárez \& Feliciano-García, 2020); y psicológicos como la depresión, ansiedad, autorregulación o déficit de auto-control empezaron a aparecer (Barraza \& Barraza, 2019; Estrada \& Mamani, 2020; Garzón \& Gil, 2017). Por su parte, Quant \& Sánchez (2012) mencionan que algunos de estos últimos comportamientos son indicios de procrastinación académica, al que definen como dilación o acto de aplazar actividades en el ámbito académico.

El ser humano a menudo pospone actividades que le parecen complicadas, estresantes o desagradables, según García \& Silva (2019). En el contexto educativo, evitar hacer tareas, la pésima gestión del tiempo para actividades, el uso de internet o de dispositivos tecnológicos son algunos factores del contexto que influencian las actitudes del estudiante (Barraza \& Barraza, 2019; García \& Silva, 2019). Por su parte Contreras et al. (2011), enfatizan que, quienes emplean su tiempo libre para jugar videojuegos, dialogar con desconocidos o buscar material para adultos, tienen altas posibilidades de aplazar actividades, ya que esto genera pereza, fatiga, falta de tiempo y, en consecuencia, la búsqueda de acciones beneficiosas a corto plazo.

A nivel internacional, la presencia de las actitudes dilatorias de los estudiantes varía según el contexto. En Turquía, entre el $23 \%$ y $27 \%$ de los estudiantes refleja actitudes procrastinadoras entre nivel medio y alto (Balkis \& Duru, 2009). En el mismo país, otra pesquisa sostiene que el 33 $\%$ mantiene conductas dilatorias siempre o casi siempre frente a los exámenes y el $30 \%$ respecto a trabajos y lecturas (Özer et al., 2009). En Jordania, un estudio sostiene que el $93 \%$ presenta niveles entre medio y alto (Mahasneh et al., 2016). En la India, el 94\% alcanza al moderado y alto (Kaur \& Rani, 2019) y en China, el 74.1\% al menos dilata una tarea; el 54.8\% lo hace con 6 y el 48.6\% aplaza los exámenes (Zhang et al., 2018).

En el contexto peruano, la revisión bibliográfica muestra algunos estudios sobre procrastinación estudiantil en distintas regiones. En Lima, el $73.2 \%$ de discentes de dos instituciones educativas de formación básica regular presenta un nivel moderado y alto de procrastinación académica, debido a que el $66.8 \%$ de ellos posterga sus actividades y el $76.4 \%$ demuestra bajos niveles de autorregulación académica (Querevalú \& Echabaudes, 2020). En la misma región, pero en el contexto universitario, los índices son un poco superiores $(80.8 \%)$ en 
cuanto a los niveles promedio y alto (Carranza \& Ramírez, 2013). En Madre de Dios, los universitarios evidencian un $60.5 \%$ de niveles alto y muy alto (Estrada \& Mamani, 2020), asimismo, en la misma región, otra indagación pero con escolares de formación básica describe que el $69.5 \%$ alcanza los mismos niveles (Estrada, 2021) y en Tarapoto, el $74.4 \%$ presenta nivel medio y alto (Villegas, 2018).

Aunque existe coincidencias porcentuales o de nivel en muchos estudios sobre procrastinación académica, las explicaciones para su análisis son distintas. Castro \& Mahamud (2017) lo asocian al conflicto que presenta el estudiante por el retraso injustificado, innecesario o irracional respecto a la finalización de tareas, además, manifiesta que esta se debe al problema de autocontrol y organización temporal. A nivel clínico, Contreras et al. (2011) exponen que es un problema relacionado con la salud neuropsíquica y social, especialmente las que se relacionan con el ámbito educativo. No obstante, el presente estudio, tomará como base los aportes de Dominguez et al., (2014), quien explica que puede entenderse desde dos factores: autorregulación académica y postergación de actividades. La primera se entiende como la acción que regula los momentos del aprendizaje, el cual por medio de la metacognición se tiene conocimiento de lo que se hace y conoce (Maite, 2012), en cambio, la segunda se asocia con aplazar tareas a pesar de que estas se puedan desarrollar al momento (Álvarez, 2010).

Debido a la coyuntura, el desarrollo de la competencia transversal 29 "Gestiona su aprendizaje de manera autónoma" (MINEDU, 2016) por parte de los alumnos carece de posibilidades de logro durante la educación remota si no se toma en cuenta o se desconoce la problemática de la dilación académica. Del mismo modo, existe una carencia de investigaciones sobre este tema en la región de Tacna, por lo tanto, es menester realizar un estudio que identifique la situación de los estudiantes del sector privado y público de la educación básica.

Es así que el estudio tiene como principal objetivo identificar el nivel de procrastinación académica de los estudiantes del nivel secundario de la provincia de Tacna en la educación a distancia. Asimismo, se pretende realizar comparaciones entre el sexo, el tipo de institución, el grado de estudios y la autoconsideración académica.

\section{Metodología}


El enfoque de investigación fue cuantitativo porque se empleó métodos y técnicas asociados a la medición de unidades, muestreo, observación y tratamiento estadístico (Ñaupas-Paitán et al., 2018), el tipo fue descriptivo, debido a que el objetivo fue identificar el nivel de procrastinación presente los estudiantes de educación secundaria, sin embargo, no se explicó los motivos o razones que generaron tales situaciones o fenómenos; asimismo, fue de corte transversal, ya que la información se recolectó en un solo momento a través de un cuestionario virtual (Bernal, 2010).

La población estuvo conformada por 1620 estudiantes del nivel secundario de cuatro instituciones educativas de primero a quinto grado de secundaria de Educación Básica Regular (EBR). Para calcular el tamaño mínimo de muestra, se consideró el $95 \%$ de confianza y $5 \%$ de error y se obtuvo como mínimo 310 estudiantes. La muestra la conformó 338 estudiantes, quienes fueron selectos mediante criterios de inclusión (Hernández \& Coello, 2008): estudiantes que pertenecen a la jurisprudencia de la DRE y UGEL Tacna, se encuentran en el nivel secundario de la EBR, están matriculados en el año lectivo 2020 en el sector público o privado, cuentan con acceso a internet y dieron su consentimiento informado.

Las características sociodemográficas (tabla 1) varían de acuerdo al sexo (F: 62.4\% - M: 37.6\%), edad (12 - 13: 34.9\%; 14 - 15: 32.8\%; 16 a más: $32.2 \%)$, grado de estudios $\left(1^{\circ}: 24 \% ; 2^{\circ}\right.$ : $16 \% ; 3^{\circ}: 13.6 \% ; 4^{\circ} 18.9 \% ; 5^{\circ}: 27.5 \%$ ), tipo de institución (pública: $59.2 \%$ y privada: $40.8 \%$ ) y, además, se tomó en cuenta si se autoconsideraban o no buenos estudiantes (Sí: 60.1\% y No: 39.9\%).

Tabla 1

Datos sociodemográficos de la muestra 


\begin{tabular}{lllc}
\hline Variable & \multicolumn{1}{c}{ Datos } & $\mathrm{N}$ & $\%$ \\
Sociodemográfica & Femenino & 211 & 62.4 \\
\hline Sexo & Masculino & 127 & 37.6 \\
& Total & 338 & 100.0 \\
Edad & $12-13$ & 118 & 34.9 \\
& $14-15$ & 111 & 32.8 \\
& 16 a más & 109 & 32.2 \\
Grado de estudios & Total & 338 & 100.0 \\
& Primero & 81 & 24.0 \\
& Segundo & 54 & 16,0 \\
& Tercero & 46 & 13.6 \\
& Cuarto & 64 & 18.9 \\
Tipo de institución & Quinto & 93 & 27.5 \\
& Total & 338 & 100.0 \\
& Pública & 200 & 59.2 \\
Autoconsideración & Privada & 138 & 40.8 \\
& Total & 338 & 100.0 \\
& Sí & 203 & 60.1 \\
& No & 135 & 39.9 \\
& Total & 338 & 100.0 \\
& & &
\end{tabular}

Fuente: Elaboración propia

El instrumento empleado fue la Escala de Procrastinación Académica (EPA) diseñado por Busko (1998), adaptado al contexto peruano por Álvarez (2010) y validado por Dominguez et al. (2014), el cual consta de 12 ítems con escala Likert de 5 puntos (Nunca: 1, Pocas veces: 2, A veces: 3, Casi siempre: 4, Siempre: 5). La validación de Dominguez et al. (2014) cataloga dos factores a los que denomina «Autorregulación académica» (se mide de forma inversa) y «Postergación de actividades» (se mide de forma directa). Con base en esto, se realizó el análisis factorial y la confiabilidad del instrumento. Los supuestos previos del análisis factorial exploratorio (AFE) indicaron que el KMO (0.957) fue superior a 0.5 y la prueba de Bartlett fue menor a 0.05, lo cual determina la continuidad del análisis (Montoya, 2007). El AFE se realizó con el método de mínimos cuadrados no ponderados con la rotación Oblimin, pero los resultados indicaron que se debía trabajar con un solo factor que explicaba el $68.145 \%$ del total de la varianza. 
Con base en el AFE, se realizó las comparaciones entre los modelos con 1 (M1) y 2 (M2) factores. De este modo, se hizo el análisis factorial confirmatorio (AFC) y se observó que, aunque las cargas factoriales de los ítems fueron similares, hubo una ligera superioridad por parte del M2. El índice de ajuste del CMIN/DF demostró que ambos modelos tuvieron puntajes adecuados (M1 $=4.471$ y M2 $=3.174)$ y un p-valor $(\mathrm{M} 1=0.000$ y M2=0.000) significativo, ya que los primeros variaron entre 3 y 5 y p fue < 0.05 (Hair et al., 2010). En el M2, el GFI fue > 0.90, el CFI > 0.95, el TLI > 0.95 y el RMSEA > 0.50 y < 0.80, es decir, evidenció índices requeridos y ajustados (Byrne, 2009; Escobedo et al., 2016; Flores et al., 2017), en cambio en el M1, ninguno de los índices alcanzaron estas exigencias. En el caso del SRMR, ambos modelos presentaron ajustes adecuados, ya que fueron < 0.05 y cercanos a 0 (Escobedo et al., 2016; Flores et al., 2017), pero el AIC del M2 fue más parsimonioso que el del M1, dado que presentó un puntaje más bajo (Escobedo et al., 2016). Por lo tanto, se tomó la decisión de emplear el M2 para el estudio (tabla 2).

Tabla 2

Comparación de cargas factoriales e índices de ajuste de los modelos 1 y 2

\begin{tabular}{|c|c|c|c|c|c|c|c|c|c|c|c|c|}
\hline \multirow{3}{*}{ Modelos } & \multicolumn{12}{|c|}{ Cargas factoriales } \\
\hline & \multicolumn{12}{|c|}{ Ítems } \\
\hline & 1 & 2 & 3 & 4 & 5 & 6 & 7 & 8 & 9 & 10 & 11 & 12 \\
\hline 1 factor & 0.79 & 0.8 & 0.81 & 0.71 & 0.77 & 0.75 & 0.85 & 0.9 & 0.82 & 0.83 & 0.84 & 0.81 \\
\hline \multirow[t]{2}{*}{2 factores } & 0.79 & 0.88 & 0.89 & 0.71 & 0.78 & 0.76 & 0.86 & 0.9 & 0.83 & 0.84 & 0.84 & 0.82 \\
\hline & \multicolumn{12}{|c|}{ Índices de ajuste } \\
\hline Modelos & \multicolumn{2}{|c|}{ CMIN/DF } & $\mathrm{P}$ & GFI & CFI & TLI & \multicolumn{2}{|c|}{ RMSEA } & \multicolumn{2}{|c|}{ SRMR } & \multicolumn{2}{|c|}{ AIC } \\
\hline 1 factor & \multicolumn{2}{|c|}{4.471} & 0.000 & 0.895 & 0.946 & 0.934 & \multicolumn{2}{|c|}{0.101} & \multicolumn{2}{|c|}{0.0325} & \multicolumn{2}{|c|}{289.45} \\
\hline 2 factores & \multicolumn{2}{|c|}{3.174} & 0.000 & 0.923 & 0.967 & 0.959 & \multicolumn{2}{|c|}{0.080} & \multicolumn{2}{|c|}{0.0322} & \multicolumn{2}{|c|}{218.232} \\
\hline
\end{tabular}

Nota: CMIN/DF: Estadístico ratio de Verosimilitud Chi-cuadrado, P: value, GFI: Índice de bondad de ajuste, CFI: Índice de ajuste comparativo, TLI: Índice Tucker-Lewis, RMSEA: Error cuadrático medio de aproximación, SRMR: Residuo estandarizado cuadrático medio, AIC: Criterio de información de Akaike Fuente: Elaboración propia

La confiabilidad de la EPA se determinó con los coeficientes Alfa de Cronbach y Omega de McDonald. La dimensión de autorregulación académica $(\alpha=0.945$ y $\omega=0.947)$ y postergación de actividades $(\alpha=0.882$ y $\omega=0.947)$ evidenciaron altos y adecuados niveles de consistencia interna y fiabilidad (Salazar \& Serpa, 2017). Asimismo, la confiabilidad de la variable general fue de 0.957 con ambos indicadores. 


\section{Procedimientos}

La recolección de información se dio entre los meses de diciembre de 2020 y enero de 2021. Se transcribió la "Escala de Procrastinación Académica (EPA)" en un Formulario de Google y se pidió permiso a los directivos de las cuatro instituciones para proceder con la aplicación. El envío del cuestionario se hizo a los docentes coordinadores del nivel secundario mediante Whatsapp, quienes después lo distribuyeron a los tutores de aula; estos últimos informaron a los padres y estudiantes sobre los objetivos de la investigación. Los participantes accedieron al formulario, leyeron las orientaciones, dieron su consentimiento voluntario e informado y lo desarrollaron. El cuestionario se cerró cuando la cantidad llegó a 338 respuestas.

El procesamiento de la base datos y codificaciones se hicieron en Excel, luego fueron exportados a los softwares: SPSS versión 25, AMOS versión 23 y Jamovi 1.2.27. Mediante tales programas se presentaron los resultados del estadístico descriptivo e inferencial. El primero se presentó en tablas de frecuencia y figuras; el segundo recurrió a la prueba de normalidad de Kolmogorov Smirnov, estadísticos de comparación de hipótesis en muestras independientes U de Mann Whitney (2) y Kruskal-Wallis (más de 2) y se calculó el tamaño del efecto. En todos los casos de trabajó con una significancia estadística de 0.05 .

\section{Criterios éticos}

El estudio se realizó con estudiantes escolares, por lo cual, los criterios éticos en la presente investigación se basan en el capítulo II: Principios éticos y capítulo IV: De la Investigación con Personas, del Código de ética de la investigación de la Universidad Nacional Jorge Basadre Grohmann - Tacna (Vicerrectorado de Investigación de la Universidad Nacional Jorge Grohmann, 2017). El fundamento se basa en la protección de la persona, el consentimiento informado y expreso, la responsabilidad científica y veracidad, la integridad científica, la justicia y el bien común y la divulgación responsable de la investigación. Asimismo, el estudio protege los derechos de los participantes, garantiza la participación libre y voluntaria de manera confidencial y anónima, además, tiene los permisos de aplicabilidad.

\section{Resultados}


Los resultados que se presentan son producto del tratamiento estadístico de la información recolectada. En la figura 1, se muestra que el $54.7 \%$ de los estudiantes postergaban regularmente sus actividades y se encontraron en nivel promedio; el $40.8 \%$ manifestó nunca o casi nunca postergar los trabajos y se ubicaron en nivel bajo y solo el $4.4 \%$ respondió que lo hacía siempre o casi siempre y se hallaron en nivel alto.

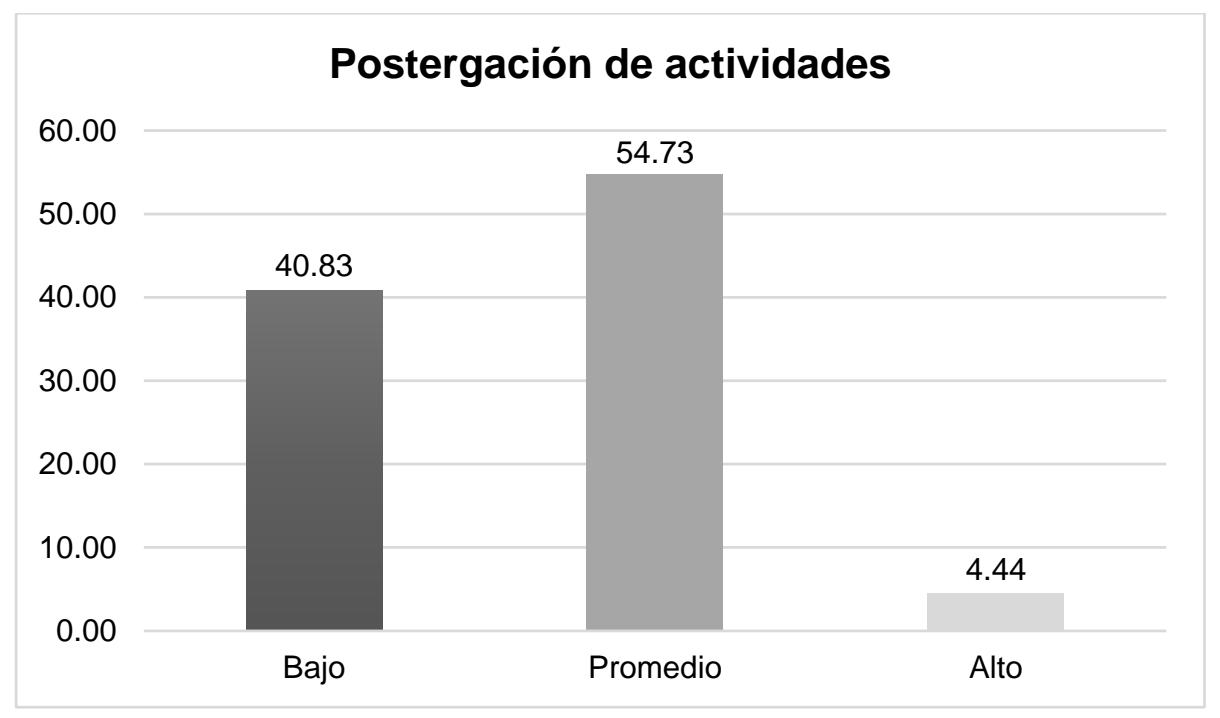

Figura 1. Postergación de actividades

En la figura 2, sobre la autorregulación académica se revela que el porcentaje de estudiantes que toma conciencia de sus deberes escolares fue de $49.70 \%$ por lo que se ubican en el nivel alto, quienes presentaron regularmente dificultades para autorregularse fueron el $25.44 \%$ y se ubicaron en el nivel promedio y cerca de la cuarta parte, es decir el $24.85 \%$, no regulaban su tiempo de estudio y se hallaron en nivel bajo de autorregulación académica. 


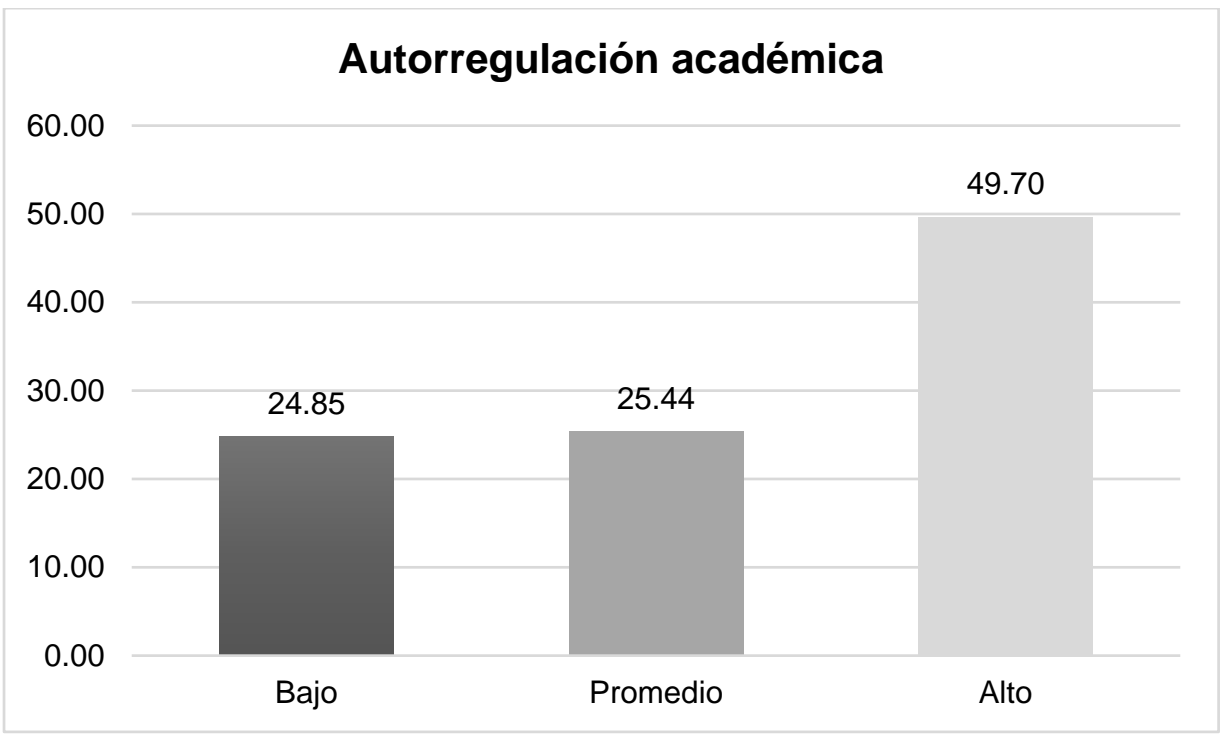

Figura 2. Autorregulación académica

Para determinar si los datos provienen o no de una distribución normal, se empleó el estadístico de Kolmogorov Smirnov y la corrección de significación de Lilliefors, debido a que la muestra es menor a 50 sujetos. Los estadísticos constataron un puntaje de 0.142 y un p-valor de $0.00<0.05$, por lo tanto, se llegó a la conclusión de que la muestra no presenta una distribución normal. De este modo, se tomó la decisión de emplear los estadísticos de prueba no paramétricos para muestras independientes de U de Mann Whitney y Kruskal-Wallis.

Se realizó una comparación entre la procrastinación existente según los datos sociodemográficos. Como sugiere Dominguez et al. (2014), esta variable no se mide directamente, sino a través de sus dos dimensiones sin un puntaje total. Por ello, se realizó la comparación según el sexo, el tipo de institución y la autoconsideración en ambas dimensiones (tabla 3). Con respecto a la postergación de actividades según el sexo, los hombres (239.96) tienden a postergar en mayor medida que las mujeres (127.09) y esta diferencia es significativa, dado que se obtuvo un valor $\mathrm{Z}$ de -10.323 , un $\mathrm{p}$-valor $=0.000$ inferior al nivel de significancia (0.05) y una magnitud de diferencia grande (0.668). Los descubrimientos se corroboran con los obtenidos en la autorregulación académica, donde las mujeres delataron mayores índices para gestionar sus actividades educativas (213.59) en comparación con los hombres (96.25); la diferencia también fue significativa porque se obtuvo un valor $\mathrm{Z}$ de -10.707 , un $\mathrm{p}$-valor $=0.000<0.05$ y un tamaño del efecto grande (0.694). En el caso del tipo de institución, se evidenció que los discentes de instituciones privadas (196.37) 
acostumbraban a postergar las actividades más que los del sector público (150.96), esta diferencia fue significativa, debido a que el valor $\mathrm{Z}$ es -4.214 y el $\mathrm{p}$-valor $=0.000<0.05$, sin embargo, el tamaño de la diferencia fue pequeño (0.269); lo cual es confirmado por los resultados de la autorregulación académica, donde las instituciones públicas (183.16) mostraron un mayor puntaje sobre los que estudiaban en las privadas (149.71), además, la diferencia fue significativa porque el valor de Z fue de -3.097 y el p-valor=0.002 <0.05; no obstante, al igual que la primera dimensión, el tamaño de diferencia fue pequeño (0.198). En el caso de quienes se autoconsideraban buenos estudiantes revelaron un bajo puntaje en la postergación de actividades (121.92) en comparación con los que no se autoconsideraban (241.04), esta diferencia resulta significativa porque $\mathrm{Z}=-11.017$ y el p-valor $=0.000<0.05$ y con una magnitud grande (0.705); además, se corrobora con los resultados de la autorregulación, donde aquellos quienes marcaron sí muestran un mayor puntaje (222.74) en comparación con los que marcaron no (89.44), la diferencia resulta también significativa debido a que $\mathrm{Z}=-12.299$ y el p-valor $=0.000<0.05$ con una gran magnitud diferenciadora (0.789) (Dominguez-Lara, 2018).

Tabla 3

Comparación de postergación de actividades y autorregulación académica según los datos sociodemográficos

\begin{tabular}{|c|c|c|c|c|c|c|}
\hline \multirow[b]{2}{*}{ Estadísticos } & \multicolumn{3}{|c|}{ Postergación de actividades } & \multicolumn{3}{|c|}{ Autorregulación académica } \\
\hline & Sexo & $\begin{array}{l}\text { Tipo de } \\
\text { institución }\end{array}$ & Autoconsideración & Sexo & $\begin{array}{l}\text { Tipo de } \\
\text { institución }\end{array}$ & Autoconsideración \\
\hline Rango promedio & $\mathrm{F}=127.09$ & Públ=150.96 & Sí=121.92 & $\mathrm{F}=213.59$ & Públ=183.16 & Sí=222.74 \\
\hline & $M=239.96$ & Priv $=196.37$ & $\mathrm{No}=241.04$ & $M=96.25$ & Priv $=149.71$ & $\mathrm{No}=89.44$ \\
\hline U de Mann- & 4450.000 & 10092.500 & 4044.500 & 4095.500 & 11069.000 & 2895.000 \\
\hline Whitney & & & & & & \\
\hline $\mathrm{Z}$ & -10.323 & -4.214 & -11.017 & -10.707 & -3.097 & -12.299 \\
\hline $\begin{array}{l}\text { Sig. } \\
\text { asintótica(bilateral) }\end{array}$ & 0.000 & 0.000 & 0.000 & 0.000 & 0.002 & 0.000 \\
\hline Tamaño del efecto & 0.668 & 0.269 & 0.705 & 0.694 & 0.198 & 0.789 \\
\hline
\end{tabular}

Nota: M: masculino, F: femenino, Públ: pública, Priv: Privada

En la tabla 4, se presenta la comparación general y por pares según el grado de estudios (primero, segundo, tercero, cuarto y quinto). Se halló que existe diferencia significativa solo entre 
el primer (194.34) y cuarto grado (140.28) de secundaria; asimismo, aunque la mayoría de comparaciones entre parejas no denotaron significatividad en las diferencias, de acuerdo con el estadístico de Kruskal-Wallis, sí existe una diferencia general $\left(\chi^{2}=12.7\right.$; p-valor=0.013 < 0.05$)$; no obstante, el tamaño o magnitud diferenciadora (0.038) no llega al mínimo necesario (0.04). Esto indica que, aunque los estudiantes de primer grado acostumbran más a posponer sus trabajos en comparación con los de cuarto, la diferencia no es grande.

En el caso de la autorregulación académica, también se observó diferencias estadísticamente significativas entre el primer grado (140.62) con el cuarto (194.21) y quinto (193.08); el p-valor en ambas diferencias fue menor (p-valor=0.008; p-valor=0.007) al nivel de significancia (0.05); asimismo, los de tercero (145.84) también mostraron diferencias estadísticamente significativas ( -valor $=0.040<0.05$; p-valor=0.029 < 0.05) en comparación con los dos últimos grados. A nivel dimensional, se evidenció diferencias $\left(\chi^{2}=19.6\right.$; $p$-valor $=0.001<$ 0.05) y una magnitud alta (0.058) que alcanza el mínimo necesario (>0.04). En este sentido, se ha identificado que la mayoría de estudiantes que cursan los grados superiores en la formación básica acostumbran a procrastinar en menor medida que quienes se inician en su formación secundaria.

Tabla 4

Postergación de actividades y autorregulación académica según el grado de estudios y comparación por pares

\begin{tabular}{|c|c|c|c|c|c|c|c|c|c|c|}
\hline \multirow[b]{2}{*}{ Pares de comparación } & \multicolumn{5}{|c|}{ Postergación de actividades } & \multicolumn{5}{|c|}{ Autorregulación académica } \\
\hline & $\mathrm{W}$ & $P$ & $\chi^{2}$ & $\mathrm{P}$ & $\varepsilon^{2}$ & $\mathrm{~W}$ & $\mathrm{P}$ & $\chi^{2}$ & $\mathrm{p}$ & $\varepsilon^{2}$ \\
\hline Primero - Segundo & -2.066 & 0.588 & & & & 1.623 & 0.781 & & & \\
\hline Primero - Tercero & -0.944 & 0.963 & & & & 0.942 & 0.964 & & & \\
\hline Primero - Cuarto & -4.554 & 0.011 & & & & 4.709 & 0.008 & & & \\
\hline Primero - Quinto & -3.214 & 0.154 & & & & 4.772 & 0.007 & & & \\
\hline Segundo - Tercero & 1.439 & 0.848 & & & & -1.039 & 0.949 & & & \\
\hline Segundo - Cuarto & -1.136 & 0.930 & 12.7 & 0.013 & 0.038 & 2.310 & 0.476 & 19.6 & 0.001 & 0.058 \\
\hline Segundo - Quinto & 0.191 & 1 & & & & 2.306 & 0.478 & & & \\
\hline Tercero - Cuarto & -3.526 & 0.092 & & & & 3.976 & 0.040 & & & \\
\hline Tercero - Quinto & -1.733 & 0.737 & & & & 4.135 & 0.029 & & & \\
\hline Cuarto - Quinto & 2.575 & 0.362 & & & & 0.094 & 1 & & & \\
\hline
\end{tabular}

Nota: (1) Los rangos promedios de cada grado según la postergación de actividades y autorregulación académica son: $1^{\circ}$ [194.34; 140.62], $2^{\circ}$ [162.92; 163.09], $3^{\circ}$ [184.63; 145.84], $4^{\circ}$ [140.28; 194.21], $5^{\circ}$ [164.31; 193.08], (2) W: valor de U de Mann Whitney, P: valor, $\chi^{2}$ : Kruskal-Wallis (chi cuadrado), $\varepsilon^{2}$ : magnitud basado en diferencias críticas 


\section{Discusión}

El objetivo general del estudio fue identificar el nivel de procrastinación académica de los estudiantes del nivel secundario de la provincia de Tacna en la educación a distancia. El primer hallazgo indica que la mayoría de estudiantes que postergan sus actividades se encuentran en nivel promedio y una minoría en el nivel alto. Los resultados discrepan algunos antecedentes que indican mayor frecuencia de alto o muy alto nivel de procrastinación (Estrada, 2021; Villegas, 2018), pero concuerdan con otros estudios realizados en contextos nacionales e internacionales (Balkis \& Duru, 2009; Carranza \& Ramírez, 2013; Mahasneh et al., 2016; Querevalú \& Echabaudes, 2020), que indican la presencia del problema en el nivel promedio, sin embargo, no descartan la existencia de actitudes dilatorias. La investigación, permite inferir que los alumnos postergan trabajos y lecturas que no son de su agrado o simplemente los aplazan de manera voluntaria porque realizan actividades que atraen más su atención como navegar por internet desde sus teléfonos móviles. El mal empleo de los smartphones como la búsqueda de información para adultos, los videojuegos o el diálogo en redes sociales generan adicción que en consecuencia puede ocasionar actitudes procrastinadoras (Contreras et al., 2011; Li et al., 2020) y con mayor razón en la educación a distancia, donde el docente presenta menor control.

Con respecto a la autorregulación académica, la mayoría de discentes se ubica en el nivel alto, pero también una cuarta parte yace en el nivel promedio y otra en inicio. El hallazgo se puede sustentar en que los encuestados cuentan con acceso a internet y apoyo de sus padres, por lo que se recomienda realizar una pesquisa comparativa considerando los factores socioeconómicos principalmente por causa del confinamiento. Se encontró algunas diferencias con otras exploraciones como el de Meza et al. (2018) y Elvira-Valdés \& Pujol (2012), los cuales informan que los estudiantes evidencian niveles moderados, lo cual es comparable con el nivel promedio. A pesar de que no se encontró estudios que revelen un nivel bajo, todavía es un problema latente, ya que su aparición genera bajos rendimientos académicos e incremento de pereza sobre todo en quienes no tienen antecedentes de calificaciones óptimas (Balkis \& Duru, 2017; Dautov, 2020). Ante esta situación, un antecedente demuestra que la autorregulación académica se relaciona directamente con el empleo de estrategias de afrontamiento (Barraza \& Barraza, 2019), debido a que abre nuevas posibilidades para el aprendizaje.

En cuanto a la variable sexo, el presente estudio halló que los hombres son quienes más postergan sus trabajos y menos autorregulan su tiempo académico en comparación con las mujeres; 
la diferencia es grande y significativa. Los hallazgos difieren con otras pesquisas que encontraron puntajes similares y no hallaron diferencias significativas entre ambos sexos (Carranza \& Ramírez, 2013; Contreras et al., 2011; Mahasneh et al., 2016) y también con aquellos cuya conclusión es que las mujeres tienen más probabilidad de diferir que los varones (Li et al., 2020; Zhang et al., 2018). Sin embargo, los descubrimientos de esta investigación concuerdan con los de otros autores (Álvarez, 2010; Balkis \& Duru, 2017; Dominguez-Lara et al., 2019; Mejia et al., 2018; Olea \& Olea, 2015; Özer et al., 2009) quienes también afirman que los niveles de procrastinación son significativamente más bajos en mujeres porque demostraron mayores índices de autorregulación y un mejor rendimiento académico, en cambio, los varones se orientaron más a postergar las tareas o trabajos escolares, alcanzaron calificaciones más bajas y poca satisfacción con la vida académica. Además, Elvira-Valdés \& Pujol (2012) explican que las mujeres emplean más estrategias de planificación, supervisión y regulación sobre su cognición para comprender los temas de clase, lo cual genera que autorregulen mejor sus aprendizajes. Además, resulta más sencillo reducir la dilación en hombres que en mujeres, ya que para los primeros se requiere mayores estrategias y métodos de autocontrol (Liu et al., 2020).

En cuanto al tipo de institución educativa, los estudiantes de instituciones privadas tienden a postergar más sus actividades y presentan menor autorregulación académica en comparación a los del sector público. Los hallazgos discrepan con los de Elvira-Valdés \& Pujol (2012) quienes concluyen que hay un menor control del tiempo de aprendizaje de alumnos de instituciones públicas en comparación al sector privado. Asimismo, Olea \& Olea (2015) no encuentran diferencias, sino puntajes similares entre ambos tipos. Al solo encontrar dos estudios interesados en comparar ambas realidades, la discusión es muy limitada, por lo tanto, es necesario aumentar la cantidad de pesquisas comparativas sobre ambos tipos de instituciones, debido a que en la virtualidad existe otros factores de influencia.

Por otro lado, los no autoconsiderados buenos estudiantes presentan mayores niveles de procrastinación con respecto a quienes sí. La magnitud de diferencia es grande y significativa. Tales revelaciones concuerdan con Li et al. (2020), cuando mencionan que los discentes que se sienten menos competentes académicamente presentan mayor tendencia enviar tardíamente sus actividades. Asimismo, Mejia et al. (2018) indica que aquellos con bajas notas, presentan mayores niveles de procrastinación, pero Dautov (2020) y Quispe et al. (2020) no lo asocian directamente a 
las notas, sino a la personalidad y actitudes motivacionales, lo cual como bien explica Hen \& Goroshit (2020), las actitudes dilatorias guardan relación con sentimientos negativos o de malestar.

En el caso del grado de estudio, existe diferencia significativa entre los estudiantes de primer y cuarto grado; los de primero postergan más las actividades. Además, la autorregulación académica de los de primer y tercer año de secundaria es inferior al de cuarto y quinto grado, por ende, se puede deducir que los grados menores tienen más acercamiento hacia actitudes procrastinadoras y no existe diferencia entre los grados superiores. Álvarez (2010) también concuerda con la paridad de resultados entre los últimos años y sustenta que se debe a la experiencia parecida para resolver situaciones de aprendizaje.

Se afirma que la literatura no brinda mucha información sobre las comparaciones de grados en educación básica, no obstante, autores como Rodríguez \& Clariana (2017) manifiestan que entre la escuela y la universidad, hay mayor aplazamiento de tareas en la primera y conforme avanza su edad desciende, debido que a la corteza prefrontal (encargada de regular la conducta, control de impulsos y concientización de consecuencias de los actos) está en proceso de desarrollo. Es por ello que se recomienda detectar estas actitudes y atenderlas de manera temprana para lograr contrarrestarlas; la mejora de la autorregulación, la autoeficacia y la regulación del esfuerzo coadyuvan a reducir tales comportamientos (Ziegler \& Opdenakker, 2018) y evitar posibles consecuencias como los altos niveles de estrés o ansiedad (Garzón \& Gil, 2017).

\section{Limitaciones del estudio}

Al ser una investigación vinculada al campo de las ciencias sociales, específicamente de la educación, es necesaria la participación de personas en el estudio, en este caso estudiantes de formación básica, elegidos mediante criterios de inclusión antes expuestos y que dieron su consentimiento informado. Asimismo, para la investigación se solicitó el permiso de los padres de manera indirecta mediante los docentes intermediarios (tutores y coordinadores) quienes colaboraron en la recolección de información, sin embargo, se sugiere hacerlo de manera directa para alcanzar una mayor muestra.

\section{Conclusiones}

Aunque los hallazgos determinan que no predomina el nivel alto en la procrastinación, medida a través de la autorregulación académica y postergación de actividades, no se descarta la 
existencia del problema en el contexto educativo, debido a que gran parte se encuentra en el nivel promedio, por lo que se requiere más estudios que corroboren los resultados. Asimismo, se ha identificado que los estudiantes hombres, de instituciones privadas, quienes no se autoconsideran buenos académicamente y de grados inferiores demuestran más probabilidad para procrastinar.

Por consiguiente, este estudio resume que, a fin de mantener las precauciones correspondientes, es necesario considerar las variables sociodemográficas en la evaluación diagnóstica para conocer la realidad de los alumnos, además, los docentes deben trabajar en conjunto con el área de psicología y realizar talleres o programas de atención estudiantil con el fin de evitar las actitudes dilatorias.

\section{Referencias}

Álvarez, Ó. (2010). Procrastinación general y académica en una muestra de estudiantes de secundaria de Lima metropolitana. Persona, 13, 159-177. https://www.redalyc.org/pdf/1471/147118212009.pdf

Balkis, M., \& Duru, E. (2009). Prevalence of academic procrastination behavior among pre-service teachers, and its relationship with demographics and individual preferences. Journal of Theory \& Practice in Education (JTPE), $5 \quad$ (1), 18-32. https://citeseerx.ist.psu.edu/viewdoc/download?doi=10.1.1.824.8098\&rep=rep1\&type=pdf

Balkis, M., \& Duru, E. (2017). Gender differences in the relationship between academic procrastination, satifaction with academic life and academic performance. Electronic Journal of Research in Educational Psychology, 15 (1), 105-125. https://doi.org/10.14204/ejrep.41.16042

Barraza, A., \& Barraza, S. (2019). Procrastinación y estrés. Análisis de su relación en alumnos de educación media superior. CPE-e. Revista de Investigación Educativa, 28, 133-151. https://doi.org/10.25009/cpue.v0i28.2602

Bernal, C. (2010). Metodología de la investigación. Bogotá: Pearson Educación.

Busko, D. A. (1998). Causes and consequences of perfectionism and procrastination: a structural equation model [Tesis de maestría, University of Guelp]. https://atrium.lib.uoguelph.ca/xmlui/handle/10214/20169

Byrne, B. M. (2009). Structural Equation Modeling with AMOS. New York: Routledge.

Carranza, R., \& Ramírez, A. (2013). Procrastinación y características demográficas asociados en estudiantes universitarios. Apuntes Universitarios, 3 (2), 95-108. 
https://www.redalyc.org/articulo.oa?id=467646127006

Castro, S., \& Mahamud, K. (2017). Procrastinación académica y adicción a internet en estudiantes universitarios de Lima Metropolitana. Avances en Psicología, 25 (2), 189-197. https://doi.org/10.33539/avpsicol.2017.v25n2.354

Contreras, H., Mori, E., Lam, N., Gil, E., Hinostroza, W., Rojas, D., Espinoza, E., Torrejón, E., \& Conspira, C. (2011). Procrastinación en el estudio: exploración del fenómeno en adolescentes escolarizados. Lima Metropolitana, Perú. Revista Peruana de Epidemiología, 15 (3), 1-5. https://www.redalyc.org/articulo.oa?id=203122771007

Dautov, D. (2020). Procrastination and laziness rates among students with different academic performance as an organizational problem. E3S Web of Conferences, 210, 1-10. https://doi.org/10.1051/e3sconf/202021018078

Dominguez-Lara, S. (2018). Effect size, a quick guide. Educacion Medica, 19 (4), 251-254. https://doi.org/10.1016/j.edumed.2017.07.002

Dominguez-Lara, S., Prada-Chapoñan, R., \& Moreta-Herrera, R. (2019). Diferencias de género en la influencia de la personalidad sobre la procrastinación académica en estudiantes universitarios peruanos. Acta colombiana de psicología, 22 (2), 125-136. https://doi.org/10.14718/acp.2019.22.2.7

Dominguez, S., Villegas, G., \& Centeno, S. (2014). Procrastinación académica: validación de una escala en una universidad privada. Liberabit, 20(2), 293-304. http://www.scielo.org.pe/pdf/liber/v20n2/a10v20n2.pdf

Elvira-Valdés, M. A., \& Pujol, L. (2012). Autorregulación y rendimiento académico en la transición secundaria-universidad. Revista Latinoamericana de Ciencias Sociales, Niñez y Juventud, 10 (1), 367-378. http://www.scielo.org.co/pdf/rlcs/v10n1/v10n1a23.pdf

Escobedo, M., Hernández, A. J., Estebané, V., \& Martínez, G. (2016). Modelos de ecuaciones estructurales: características, fases, construcción, aplicación y resultados. Revista Ciencia y Trabajo, 18(55), 16-22. https://doi.org/10.4067/S0718-24492016000100004

Estrada, E. G. (2021). Autoeficacia y procrastinación académica en estudiantes del séptimo ciclo de educación básica regular. Horizonte de La Ciencia, 11 (20), 195-205. https://doi.org/10.26490/uncp.horizonteciencia.2021.20.777

Estrada, E. G., \& Mamani, H. J. (2020). Procrastinación académica y ansiedad en estudiantes universitarios de Madre de Dios, Perú. Apuntes Universitarios, 10 (4), 322-337. 
https://doi.org/10.17162/au.v10i4.517

Flores, O., Lajo, Y., Zevallos, A., Rondán, P. L., Lizaraso, F., \& Jorquiera, T. (2017). Análisis psicométrico de un cuestionario para medir el ambiente educativo en una muestra de estudiantes de medicina en Perú. Revista Peruana de Medicina Experimental y Salud Publica, 34 (2), 255-260. https://doi.org/10.17843/rpmesp.2017.342.2642

García, V., \& Silva, M. (2019). Procrastinación académica entre estudiantes de cursos en línea. Validación de un cuestionario. Apertura, $11 \quad$ (2), 122-137. https://doi.org/10.32870/Ap.v11n2.1673

Garzón, A., \& Gil, J. (2017). El papel de la procrastinación académica como factor de la deserción universitaria. Revista Complutense de Educación, 28 (1), 307-324. https://doi.org/10.5209/rev_RCED.2017.v28.n1.49682

Hair, J. F., Black, W. C., Babin, B. J., \& Anderson, E. (2010). Multivariate Data Analysis. Londres: Pearson Education.

Hen, M., \& Goroshit, M. (2020). The effects of decisional and academic procrastination on students' feelings toward academic procrastination. Current Psychology, 39 (2), 556-563. https://doi.org/10.1007/s12144-017-9777-3

Hernández, R., \& Coello, S. (2008). El paradigma cuantitativo de la investigación científica. La Habana: Editorial Universitaria.

Kaur, K., \& Rani, M. (2019). Demographic study on academic procrastination among secondary school students. International Journal of Education, 11, 73-81. http://ijoe.vidyapublications.com/Issues/Vol11/08_Vol.11.pdf

Li, L., Gao, H., \& Xu, Y. (2020). The mediating and buffering effect of academic self-efficacy on the relationship between smartphone addiction and academic procrastination. Computers and Education, 159. https://doi.org/10.1016/j.compedu.2020.104001

Liu, G., Cheng, G., Hu, J., Pan, Y., \& Zhao, S. (2020). Academic Self-Efficacy and Postgraduate Procrastination: A Moderated Mediation Model. Frontiers in Psychology, 11, 1-9. https://doi.org/10.3389/fpsyg.2020.01752

Mahasneh, A. M., Bataineh, O. T., \& Al-Zoubi, Z. H. (2016). The relationship between academic procrastination and parenting styles among jordanian undergraduate university students. The Open Psychology Journal, 9 (1), 25-34. https://doi.org/10.2174/1874350101609010025

Maite, G. (2012). La autorregulación académica como variable explicativa de los procesos de 
aprendizaje universitario. Profesorado, $16 \quad$ (1), 203-221. https://www.redalyc.org/pdf/567/56724377012.pdf

Mejia, C. R., Ruiz-Urbina, F. N., Benites-Gamboa, D., \& Pereda-Castro, W. (2018). Factores académicos asociados a la procrastinación. Revista Cubana de Medicina General Integral, 34 (3), 61-70. http://www.revmgi.sld.cu/index.php/mgi/article/view/954

Meza, J. M., De la Rosa, A., Rivera, J., \& Santiago, E. (2018). Evaluación de autorregulacion académica en estudiantes de psicología en modalidad en línea. Voces de la educación, 3 (6), $126-141$.

https://www.revista.vocesdelaeducacion.com.mx/index.php/voces/article/view/125/112

Ministerio de Educación (MINEDU). (2016). Programa curricular de Educación Secundaria (p. 259). http://repositorio.minedu.gob.pe/handle/123456789/4550

Montoya, O. (2007). Aplicación del análisis factorial a la investigación de mercados. Caso de estudio. Scientia et Technica, 3 (35), 281-286. https://doi.org/10.22517/23447214.5443

Ñaupas, H., Valdivia, M. R., Palacios, J. J., \& Romero, H. E. (2018). Metodología de la investigación. Cuantitativa - Cualitativa y Redacción de la Tesis. Bogotá: Ediciones de la U.

Olea, M. T., \& Olea, A. N. (2015). Perceptiveness and sense impression of procrastination across correlates. International Research Journal of Social Sciences, 4 (1), 37-43. http://www.isca.in/IJSS/Archive/v4/i1/6.ISCA-IRJSS-2014-271.pdf

Organización de las Naciones Unidas para la Educación la Ciencia y la Cultura [UNESCO]. (2020). COVID 19 - Impact on Education. https://en.unesco.org/covid19/educationresponse

Özer, B. U., Demir, A., \& Ferrari, J. R. (2009). Exploring academic procrastination among turkish students: Possible gender differences in prevalence and reasons. Journal of Social Psychology, 149(2), 241-257. https://doi.org/10.3200/SOCP.149.2.241-257

Quant, D., \& Sánchez, A. (2012). Procrastinación, procrastinación académica: concepto e implicaciones. Revista Vanguardia Psicológica Clínica Teórica y Práctica, 57 (1), 45-59. https://dialnet.unirioja.es/servlet/articulo?codigo=4815146

Querevalú, F., \& Echabaudes, R. (2020). Procrastinación académica y ansiedad frente a los exámenes en estudiantes de 3 a 5 del nivel secundario en colegios de Lima. Revista Científica de Ciencias de La Salud, 13(1), 79-87. https://doi.org/10.17162/rccs.v13i1.1350

Quispe, L., Araujo, R., García, J., García, Y., Sprock, A., \& Villalba, K. (2020). Relationship between academic procrastination and attributions of achievement motivation. International 
Journal of Learning, Teaching and Educational Research, 19 (1), 188-205. https://doi.org/10.26803/ijlter.19.1.11

Resolución Viceministerial Nº79-2020-MINEDU [Ministerio de Educación]. 12 de marzo de 2020. Diario El Peruano. https://cutt.ly/Mx23jfu

Resolución Ministerial 160-2020-MINEDU [Ministerio de Educación]. 1 de abril de 2020. Diario El Peruano. https://cutt.ly/5x23AB7

Rodríguez, A., \& Clariana, M. (2017). Procrastinación en estudiantes universitarios: su relación con la edad y el curso académico. Revista Colombiana de Psicologia, 26 (1), 45-60. https://doi.org/10.15446/rcp.v26n1.53572

Salazar, C., \& Serpa, A. (2017). Análisis confirmatorio y coeficiente Omega como propiedades psicométricas del instrumento Clima Laboral de Sonia Palma. Revista de Investigación En Psicología, 20 (2), 377-388. https://doi.org/10.15381/rinvp.v20i2.14047

Suárez, A., \& Feliciano-García, L. (2020). Influencia del perfil de procrastinación activa en el rendimiento académico del alumnado de ciencias de la educación. Bordon. Revista de Pedagogia, 72 (3), 157-170. https://doi.org/10.13042/Bordon.2020.73642

Vicerrectorado de Investigación de la Universidad Nacional Jorge Grohmann. (2017). Código de ética para la investigación de la Universidad Nacional Jorge Basadre Grohmann - Tacna. Tacna: Universidad Nacional Jorge Basadre Grohmann. http://unjbg.edu.pe/investigacion/pdf/20180216-codigo-etica.pdf

Villegas, M. (2018). Procrastinación y autoeficacia académica en estudiantes del nivel secundario del Colegio Simón Bolívar de Tarapoto. Revista Cientifi-K, 6 (2), 116-122. https://doi.org/10.18050/cientifi-k.v6n2a2.2018

Zhang, Y., Dong, S., Fang, W., Chai, X., Mei, J., \& Fan, X. (2018). Self-efficacy for self-regulation and fear of failure as mediators between self-esteem and academic procrastination among undergraduates in health professions. Advances in Health Sciences Education, 23 (4), 817830. https://doi.org/10.1007/s10459-018-9832-3

Ziegler, N., \& Opdenakker, M. C. (2018). The development of academic procrastination in firstyear secondary education students: The link with metacognitive self-regulation, self-efficacy, and effort regulation. Learning and Individual Differences, 64, 71-82. https://doi.org/10.1016/j.lindif.2018.04.009 